\title{
As Variações dos Intervalos de Tempo entre as Fases Principais da Lua
}

The variations within main phases of the Moon time intervals

\author{
Fernando Lang da Silveira \\ Instituto de Física, Universidade Federal do Rio Grande do Sul \\ Caixa Postal 15051, 91501-970, Porto Alegre, RS, Brasil \\ Endereço eletrônico: lang@if.ufrgs.br
}

Recebido em 16 de Agosto de 2001. Aceito em 01 de Outubro 2001.

\begin{abstract}
Os intervalos de tempo entre as sucessivas fases principais da Lua (Nova, Quarto Crescente, Cheia e Quarto Minguante) não são idênticos e apresentam pronunciadas variações ao longo do tempo. Neste trabalho é elaborada uma explicação qualitativa para essas "anomalias". É também apresentado um conjunto de equações que permite o cálculo do momento em que cada fase principal ocorre. As equações foram geradas a partir de tabelas de lunações por procedimentos de regressão não-linear; com elas é possível se estimar os momentos das fases principais com um erro padrão (desvio padrão da estimativa) de $20 \mathrm{~min}$. As tabelas de lunações foram obtidas junto ao Observatório Nacional do Brasil e se referem ao período de 1940 a 2020.
\end{abstract}

The time intervals within each main phase of the Moon (new moon, first-quarter, full-moon and last-quarter) are not identical. In fact they happen to vary pronouncedly on time. A qualitative explanation for these "anomalies" is outlined at the beginnig of this text. Additionally the problem is solved using a set of equations worked out by non-linear regression procedures. The equations allow to calculate date and hour of a main phase occurence within a standard error of $20 \mathrm{~min}$. The data were extracted from lunation-tables obtained at Observatório Nacional do Brasil for the period of $1940-2020$.

\section{Introdução}

As quatro principais fases da Lua (Nova, Quarto Crescente, Cheia e Quarto Minguante) ocorrem nessa ordem durante um mês sinódico ou lunação, cuja duração é de aproximadamente 29,5 dias. Então, poder-se-ia pensar que o intervalo de tempo entre duas fases consecutivas da Lua é um quarto de 29,5 dias. Contudo, a consulta a um calendário com as datas das fases principais da Lua revela que tal idéia seria errada. O número de dias entre fases consecutivas é em sua maioria 7 ou 8 , mas também ocorrem intervalos de até 9 dias ou de apenas 6 dias. Por exemplo, em 5 de junho de 2001 ocorre uma Cheia que é seguida 9 dias depois (14 de junho de 2001) pela Quarto Minguante; em 12 de agosto de 2001 acontece uma Quarto Minguante que é sucedida após 6 dias (18 de agosto) pela Nova. Entre 2000 e 2002 ocorrem, entre as fases principais sucessivas, apenas 5 intervalos de 6 dias e 4 de 9 dias. Dos restantes 90 intervalos, 55 são de
7 dias e 35 são de 8 dias. Se fizermos um levantamento do número de dias entre as fases principais consecutivas durante um grande período de tempo (entre $1940 \mathrm{e}$ 2020), verificaremos que $48,0 \%$ desses intervalos são de 7 dias, 40,9\% são de 8 dias, somente $8,3 \%$ são de 6 dias e apenas 2,8\% são de 9 dias. A Fig.(1) mostra, em dias inteiros, o tempo que, entre 2000 e 2002, separa cada fase principal da Lua da fase seguinte.

Observa-se na Fig.(1) que para uma específica fase principal, por exemplo Nova, o tempo para a fase que lhe sucede é variável $(6,7,8$ ou 9 dias).

A explicação para a existência das diferentes fases da Lua já era conhecida desde a Antiguidade. Aristóteles (384 - 322 A.C.), em sua obra Analítica Posterior, já destacava que a Lua não possui luz própria e que a sua face brilhante é a face voltada para o Sol (Losee, 1993). Como a Lua gira em torno da Terra, sua aparência para um observador terrestre dependerá da posição relativa Sol -Lua-Terra. 


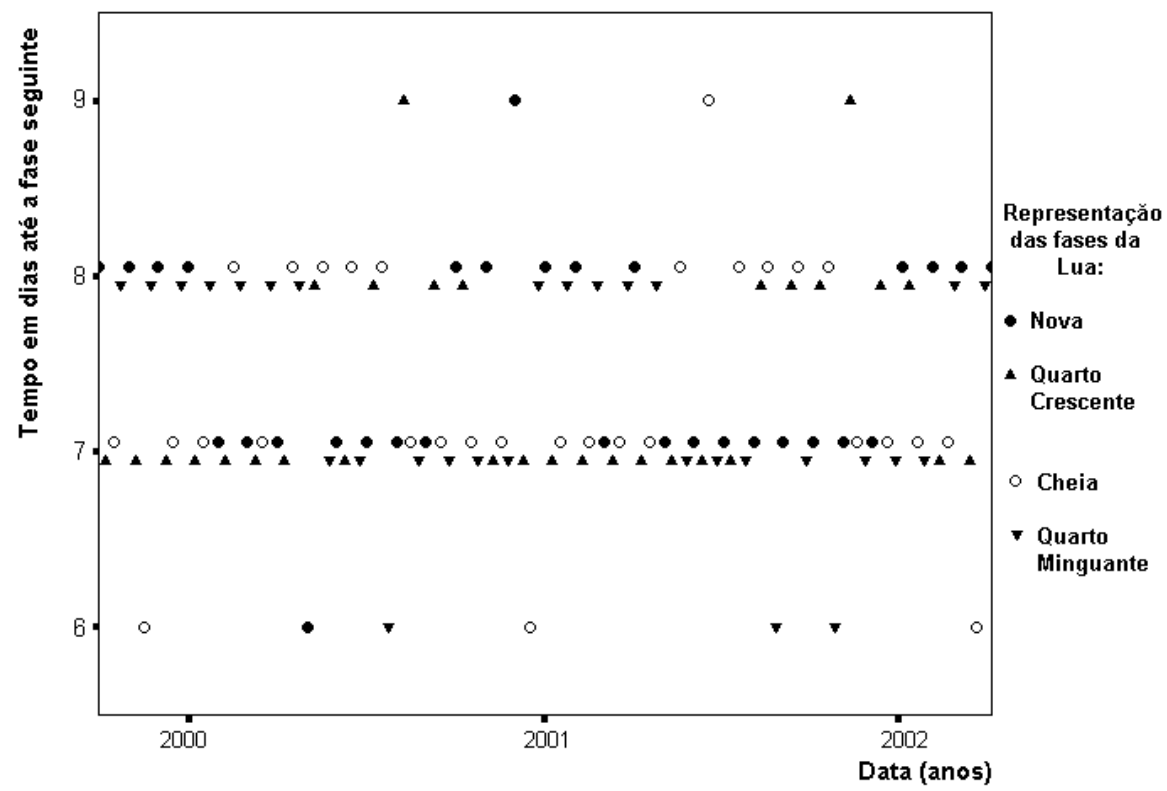

Figura 1. Dias inteiros que transcorrem entre uma fase principal da Lua e a fase seguinte no período de 2000 a 2002.

\section{Uma explicação para as fases da Lua}

Na Fig.(2) é apresentado um típico diagrama utilizado para explicar as fases da Lua, representando-a em movimento de translação circular uniforme em torno da Terra; a parte da Terra que ali aparece é o hemisfério norte $^{1}$, que gira no sentido anti-horário (mesmo sentido da translação da Lua em torno da Terra). O Sol, muito distante, ilumina a região do sistema Terra-Lua com raios luminosos praticamente paralelos entre si, mas não exatamente paralelos ao plano da órbita da Lua.

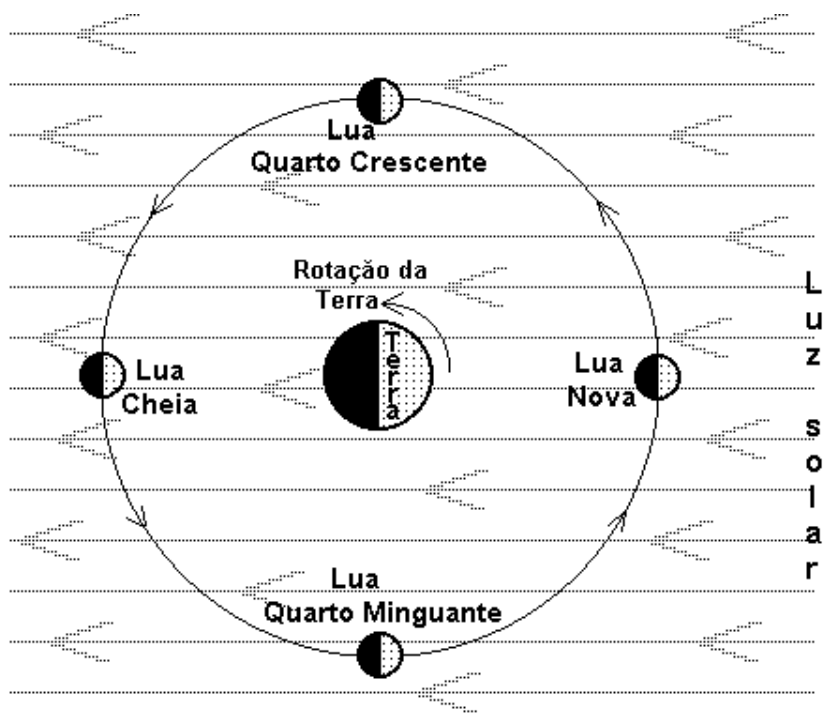

Figura 2. Lua em órbita circular em torno da Terra.
O plano da órbita da Lua em torno da Terra se inclina aproximadamente 5 graus em relação ao plano da órbita da Terra em torno do Sol (eclíptica), conforme representa a Fig.(3) (para que esse ângulo possa ser representado, a figura o exagera). Caso esses dois planos coincidissem, a cada Nova ocorreria um eclipse do Sol e a cada Cheia ocorreria um eclipse da Lua. Os eclipses somente são possíveis quando a Lua, na fase Nova ou Cheia, estiver próxima a um dos dois pontos de interseção da sua órbita com o plano da eclíptica (esses pontos são denominados nodos); a cada ano ocorrem no mínimo dois e no máximo sete eclipses(Mourão,1993).

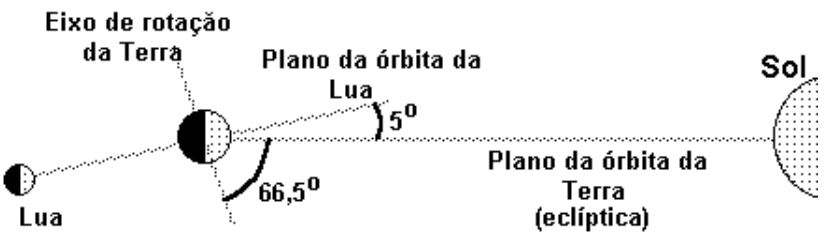

Figura 3. Plano da órbita da Lua e a eclíptica.

A Fig.(4) explicita melhor as posições relativas SolLua-Terra em cada uma das quatro fases principais. É importante destacar que, nas figuras deste trabalho, os diâmetros dos três corpos, bem como as distâncias entre seus centros, não estão representados em escala. Uma Nova ou Cheia ocorre quando, ignorando-se o fato de

\footnotetext{
${ }^{1} \mathrm{Na}$ verdade o eixo de rotação da Terra está inclinado cerca de $66,5^{\circ}$ em relação ao plano de sua órbita em torno do Sol (eclíptica). Desta forma, a região da Terra vista na figura é em sua maior parte o hemisfério norte, mas também aparece uma pequena parcela do hemisfério sul.
} 
que o plano da órbita lunar não coincide com a eclíptica, os três corpos estão alinhados. A fase será Quarto Crescente ou Quarto Minguante quando o ângulo Sol-LuaTerra for $90^{\circ}$.
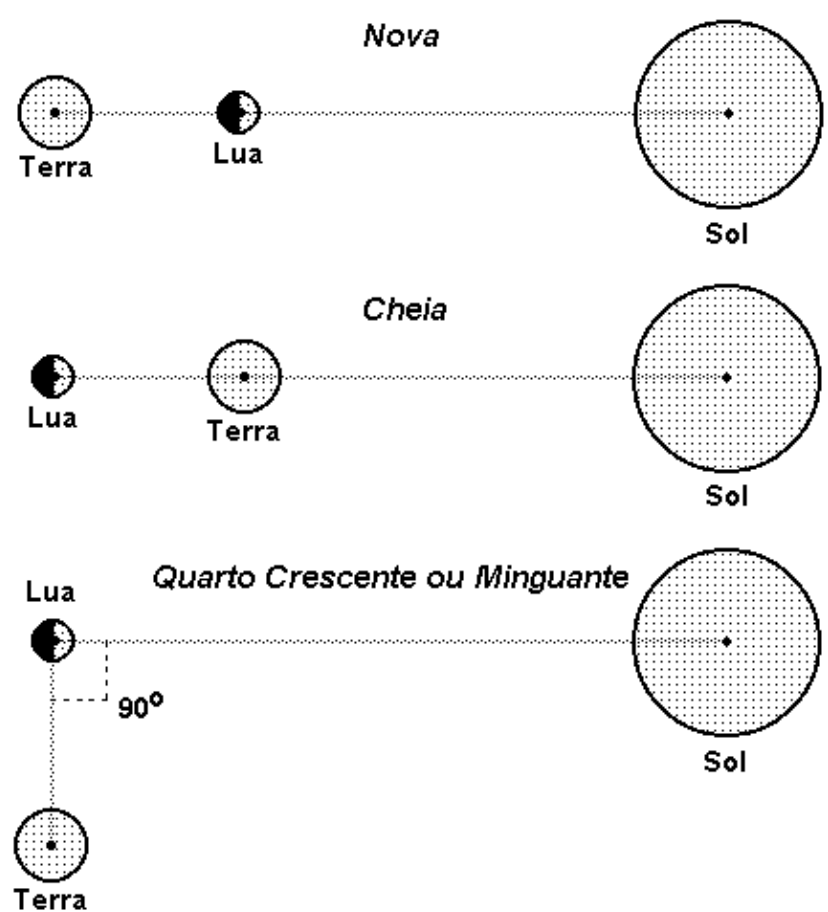

Figura 4. Posição relativa Sol-Lua-Terra para as quatro fases principais da Lua.

Cada posição relativa está associada a uma específica posição da Lua em sua órbita. O horário em que ocorre uma fase principal é muito bem conhecido e pode ser encontrado em uma tabela de lunações ${ }^{2}$ (essas tabelas adotam precisão de minuto; já a maioria dos calendários comuns assinalam apenas o dia em que ocorre uma fase principal). Uma consulta à tabela para 2002, produzida pelo Observatório Nacional, revela que a primeira Lua Nova de 2002 será em 13 de janeiro, às 10 h 29 min pelo horário normal de Brasília.

A Fig.(5) constitui-se em uma representação mais precisa dos intervalos de tempo que separam uma fase principal da subseqüente. Percebe-se que esses intervalos variam entre aproximadamente 6,5 dias e 8,3 dias; em média transcorrem 7,38 dias entre uma fase principal e a subseqüente.

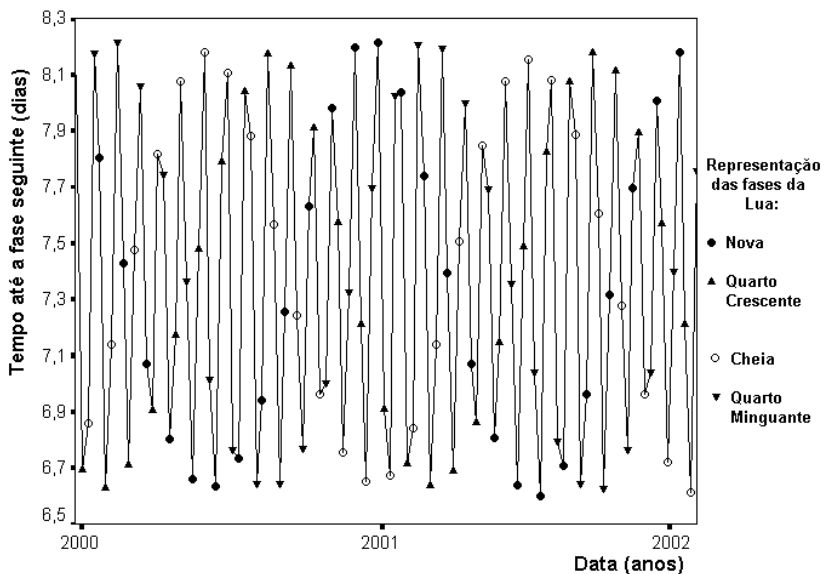

Figura 5. Tempo decorrido entre uma fase principal da Lua e a fase seguinte no período de 2000 a 2002 .

O modelo adotado na Fig.(2) não consegue explicar as variações do intervalo de tempo entre duas fases principais consecutivas; da representação feita naquela figura decorre que o intervalo de tempo entre duas fases principais consecutivas deveria ser sempre o mesmo, já que a Lua se encontra em movimento circular uniforme em torno da Terra.

No presente trabalho pretende-se descrever com mais precisão o movimento da Lua, possibilitando assim uma explicação qualitativa para a irregularidade dos intervalos de tempo entre as fases principais. No final serão apresentadas algumas equações que auxiliam a efetuar o cálculo do momento em que cada uma das quatro fases ocorrem. Todos os dados sobre os momentos em que ocorrem as fases principais da Lua foram retirados de tabelas de lunações de 1940 a 2020, produzidas pelo Observatório Nacional ${ }^{3}$.

\section{Aprimorando a explicação: a órbita do sistema Terra- Lua em torno do Sol e a órbita da Lua em torno da Terra são elípticas}

A primeira correção importante no modelo apresentado na Fig.(2) é que a distância Terra-Lua é variável, sendo a órbita lunar em relação à Terra aproximadamente elíptica ${ }^{4}$. A excentricidade da órbita lunar não é desprezível; a distância média entre o centro da Terra e o

\footnotetext{
${ }^{2}$ Uma tabela de lunações, para um determinado ano, fornece, para qualquer das fases principais da Lua, a data do evento (mês e dia) e o horário com precisão de minuto. O cálculo de tais tabelas não é simples, pois a órbita da Lua é irregular. Tais irregularidades ocorrem devido às influências do Sol e dos planetas, à não esfericidade da Terra e aos efeitos de maré (cálculos precisos da órbita da Lua envolvem mais de uma centena de termos). Neste trabalho utilizamos tabelas de lunações geradas pelo Observatório Nacional do Brasil.

${ }^{3} \mathrm{O}$ leitor interessado poderá facilmente obter tabelas de lunações (e outras tantas informações astrônomicas) acessando o endereço eletrônico do Observatório Nacional (www.on.br). Existem inúmeros outros "sites" de Astronomia que podem ser consultados, entre eles, www.usno.navy.mil, www.if.ufrgs.br/ast.

${ }^{4}$ Caso o sistema Terra-Lua consistisse de dois corpos com distribuição de massa esférica e não interagisse com qualquer outro corpo (Sol, planetas e demais constituintes do sistema solar), o movimento relativo Terra-Lua seria exatamente elíptico, sempre com o mesmo período.
} 
centro da Lua é $384.000 \mathrm{~km}$ (60,3 raios terrestres), variando entre $356.800 \mathrm{~km}$ (56,0 raios terrestres) no perigeu e $406.400 \mathrm{~km}$ (63,8 raios terrestres) no apogeu (Oliveira Filho e Saraiva, 1997).

O período sideral da Lua (intervalo de tempo em que a Lua descreve uma volta em torno da Terra no sistema de referência das estrelas fixas) é aproximadamente $27,32 \operatorname{dias}^{5}$. O tempo entre duas fases iguais consecutivas (por exemplo, duas Novas consecutivas), denominado de período sinódico da Lua, é aproximadamente 29,53 dias. Para se compreender a diferença entre esses dois períodos, deve-se ter em conta que enquanto a Lua descreve uma volta em torno da Terra, o centro de massa do sistema Terra-Lua ${ }^{6}$ se translada em torno do Sol em uma órbita aproximadamente circular ${ }^{7}$. A Fig.(6) representa essa situação (as dimensões dos três corpos, as distâncias entre os seus centros e a excentricidade da órbita da Lua não estão em escala; a distância média Terra-Sol é cerca de 394 vezes a distância média Terra-Lua).

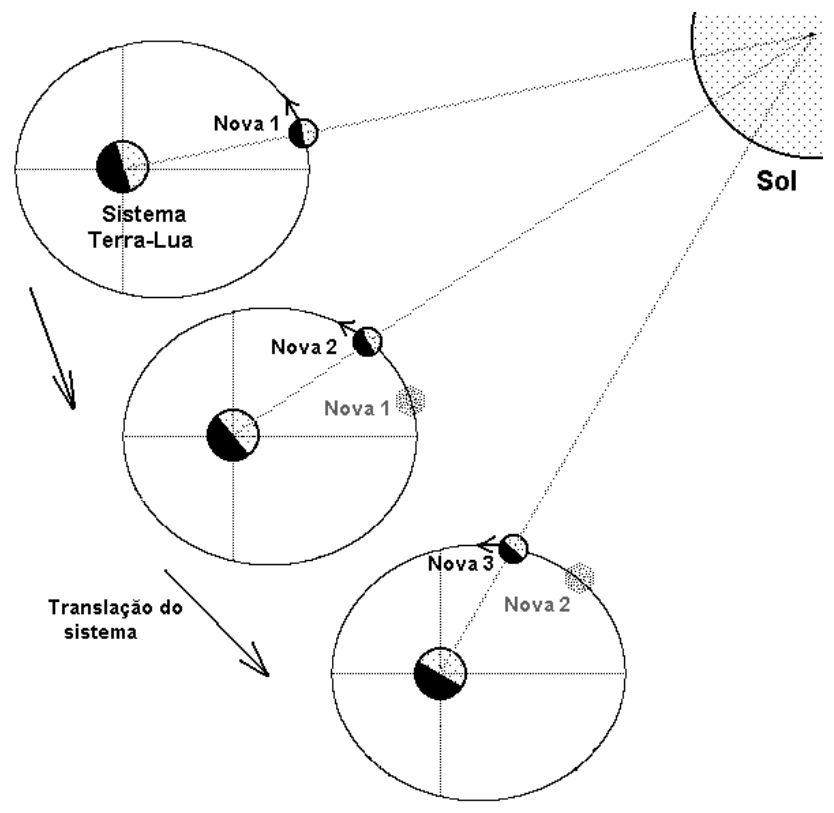

Figura 6. Representação de três Novas consecutivas.

A Fig.(6) nos ajuda a perceber que, após transcorrer um mês sinódico, isto é, no período entre duas Novas consecutivas, a Lua se translada mais do que uma volta completa em torno da Terra; assim, o período sinódico da Lua acaba sendo maior do que o seu período sideral.

Devido à excentricidade da órbita do sistema TerraLua em torno do Sol e da órbita da Lua em torno da Terra, as velocidades angulares desses dois movimentos são variáveis ${ }^{8}$. Como a duração do mês sinódico depende das duas velocidades angulares, o período sinódico da Lua não poderia ser constante.

A Fig.(7) mostra como a duração do mês sinódico (de Nova a Nova) varia entre 1984 e 2006.

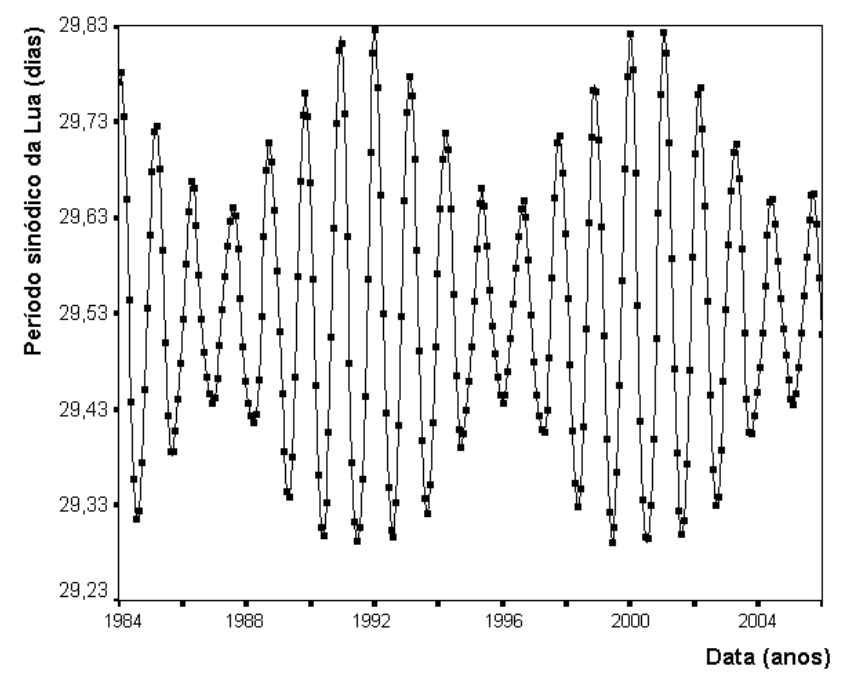

Figura 7. Variação do período sinódico da Lua entre 1984 e 2006.

O período sinódico da Lua é em média 29,53 dias, variando entre 29,3 e 29,8 dias. As causas dessas variações, além das já referidas acima, são diversas (mais adiante retomaremos essa questão).

A excentricidade da órbita da Lua em torno da Terra também é responsável por variações no intervalo de tempo que separa duas fases principais consecutivas. Na Fig.(8) é possível perceber que em um dado mês lunar, os comprimentos das trajetórias entre as fases principais consecutivas não são iguais ${ }^{9}$; também a Fig.(8) indica que em meses diversos, o comprimento da trajetória entre as mesmas duas fases principais consecutivas (por exemplo, entre Nova e Quarto Crescente) é diferente. Assim, os intervalos de tempo associados às passagens entre as fases principais (que dependem não

\footnotetext{
${ }^{5}$ Mais precisamente, o período sideral da Lua é em média 27,32166 dias, variando em até 7 horas devido às perturbaçôes que o sistema Terra-Lua sofre (Payne-Gaposchkin e Haramundanis; 1970).

${ }^{6}$ A massa da Lua é aproximadamente $1 / 81$ da massa da Terra. O centro de massa do sistema Terra-Lua situa-se na linha que une o centro dos dois corpos, em média a 0,74 raios terrestres do centro da Terra. Assim sendo, o centro de massa do sistema encontra-se dentro da Terra.

${ }^{7}$ A excentricidade da órbita do sistema Terra- Lua em torno do Sol é 0,017 ; ao periélio corresponde uma distância de 147,1 x $10^{6}$ $\mathrm{km}$ e ao afélio $152,1 \times 10^{6} \mathrm{~km}$. A excentricidade da órbita da Lua em torno da Terra é 0,055, portanto cerca de três vezes a do sistema Terra-Lua em torno do Sol.

${ }^{8}$ A Mecânica Newtoniana (conservação da quantidade de movimento angular) permite demonstrar que quando diminui o raio da órbita, aumenta a velocidade angular.

${ }^{9}$ Por simplicidade, na Fig.(8), não está sendo considerada a translação do sistema Terra? Lua durante o mês sinódico. Esta translação determina que o deslocamento angular da Lua em relação à Terra entre duas fases principais consecutivas seja maior do que $90^{\circ}$ (aproximadamente $97^{\circ}$ ).
} 
apenas do comprimento das trajetórias mas também da velocidade linear ${ }^{10}$ em cada ponto da trajetória), não são iguais ao longo do mesmo mês lunar, variando também de um mês para outro (vide os gráficos das Figs.(1) e (5)).

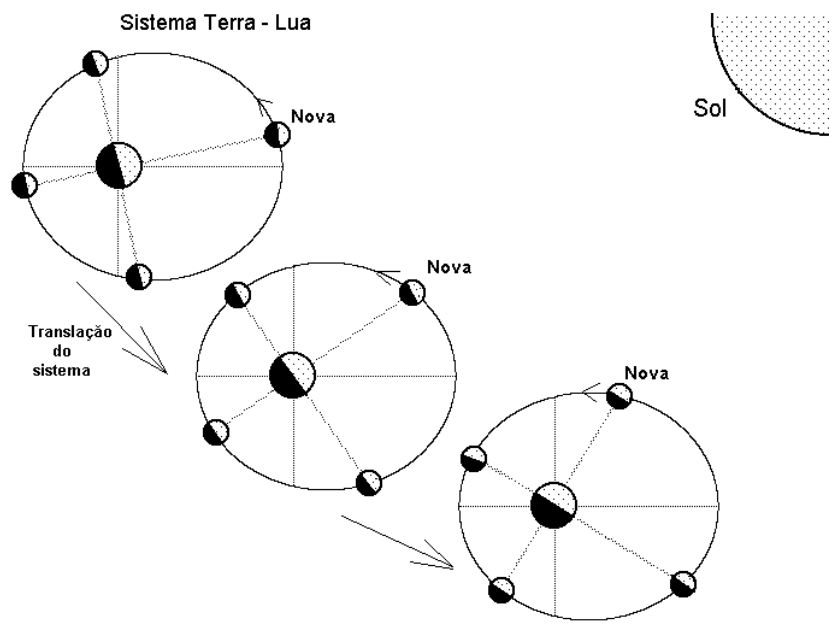

Figura 8. Representação das fases principais da Lua em diferentes meses lunares.

\section{Perturbações sofridas pelo sistema Terra-Lua}

O movimento da Lua em relação à Terra depende preponderantemente da interação gravitacional entre esses dois corpos. Entretanto, tal movimento sofre perturbações devidas às ações gravitacionais dos demais corpos do sistema solar. As perturbações mais importantes são aquelas produzidas pelo Sol porque o campo gravitacional do Sol sobre o sistema Terra-Lua não é uniforme. Conforme Silveira e Axt (p. 232-233, 2000):

O campo gravitacional externo ao sistema Terra-Lua, produzido pelo Sol, não é rigorosamente uniforme pois as dimensões do sistema não são desprezíveis frente à distância que o separa do Sol. Na Terra, cujo raio é cerca de sessenta vezes menor do que a distância Terra-Lua, uma manifestação da não-uniformidade do campo gravitacional do Sol ocorre nos efeitos de maré observados nos oceanos: o Sol, que atrai a Terra com uma força cerca de 200 vezes a força de atração da Lua, contribui para os efeitos de maré com forças de maré que perfazem $46 \%$ das forças de maré lunares (Marion e Thornton, 1995). As variações do campo gravitacional produzido sobre o sistema pelo Sol afetam o movimento relativo Lua-Terra, determinando pequenas perturbações na trajetória da Lua. Caso o campo gravitacional externo ao sistema Terra-Lua fosse uniforme, o movimento relativo Lua-Terra dependeria apenas da atração gravitacional mútua entre o planeta e o seu satélite; como não o é, esse movimento depende também do campo externo. De fato, a não-uniformidade do campo gravitacional externo não se deve apenas ao Sol; os demais planetas do sistema solar também contribuem, produzindo outras tantas pequenas perturbações no movimento relativo Lua-Terra.

Segundo as autoras Payne-Gaposchkin e Haramundanis (p. 169, 1970) "o movimento da Lua pode ser decomposto em aproximadamente 150 termos periódicos principais ao longo da eclíptica, e outros tantos na direção perpendicular a ela". Elas enumeram as 8 perturbações mais importantes sofridas pelo sistema TerraLua, sendo algumas delas conhecidas anteriormente a Newton (século XVII), até mesmo na Antiguidade.

Em conseqüência das perturbações, o movimento da Lua relativamente à Terra é irregular; a duração do mês sinódico, bem como os intervalos de tempo entre as fases principais consecutivas, são afetados por tais irregularidades. Para a análise que será apresentada nas seções 5 e 6 , é importante destacar três das 8 perturbações principais: a variação, a equação anual e a rotação da linha de apside.

Segundo Payne-Gaposchkin e Haramundanis (p. 168, 1970):

A variação é um efeito que faz com que a Nova $e$ a Cheia ocorram mais cedo, $e$ a Quarto Crescente e a Quarto Minguante mais tarde no ciclo.

A equação anual é um resultado da mudança da força perturbadora do Sol quando a Terra viaja em sua órbita elíptica, e a distância que a separa do Sol se modifica.

$A$ variação e a equação anual foram observa $\overline{d a s ~ p e l a ~ p r i m e i r a ~ v e z ~ p o r ~ T y c h o ~ B r a h e ~}$ por volta de 1600.

A linha de apside é a linha que passa pelos pontos de máxima aproximação da Lua em relação à Terra (perigeu) e máximo afastamento de Lua em relação à Terra (apogeu); ou seja, a linha de apside constitui-se no semi-eixo maior da elipse que representa a órbita da Lua em relação ao centro de massa do sistema TerraLua. O centro de massa do sistema Terra-Lua, que está em um dos focos dessa elipse, translada-se em torno do Sol em uma órbita aproximadamente circular (vide as Figs. (6) e (8)). Concomitante com esse movimento, a

\footnotetext{
${ }^{10} \mathrm{~A}$ velocidade linear da Lua em relação à Terra é máxima no perigeu e mínima no apogeu, variando de um ponto para outro da trajetória.
} 
elipse gira lentamente (o que não está representado nas Figs. (6) e (8)); essa rotação perfaz um ângulo de aproximadamente $40^{\circ}$ por ano, de tal forma que em aproximadamente 9 anos ocorre uma rotação completa da linha de apside. As Figs. (9(a)) e (9(b)), que ajudam a imaginar o sistema Terra-Lua em dois momentos separados de um ano, representam tal rotação (novamente é importante destacar que as dimensões dos três corpos e as distâncias entre eles não estão em escala; também a excentricidade da órbita da Lua está exagerada); a Fig.(9(b)) apresenta o ângulo rotado em um ano, isto é, o ângulo entre a linha de apside (linha que passa por A e P) nessa figura com a linha de apside representada na Fig.(9(a)), um ano antes.

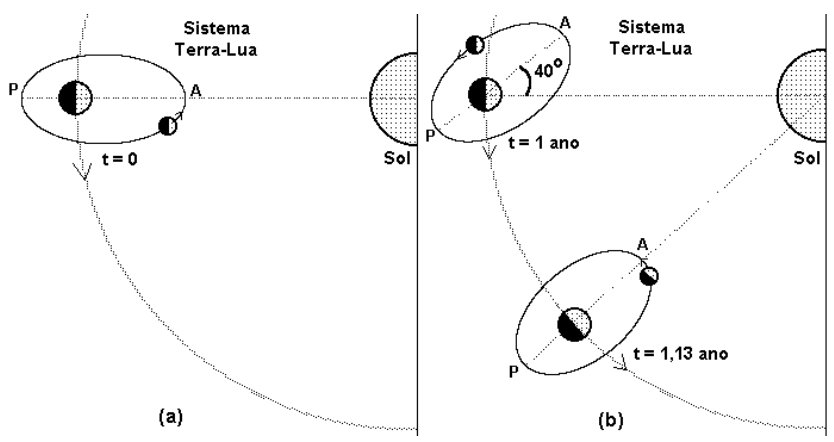

Figura 9. Representação da rotação da linha de apside: (a) em $\mathrm{t}=0$ e (b) em $\mathrm{t}=1$ ano e $\mathrm{t}=1,13$ ano.

A Fig.(9(b)) também apresenta o tempo para que a linha de apside volte a coincidir em orientação com a linha Terra-Sol (direção de onde vem a luz solar); esse tempo é aproximadamente 1,13 ano ou 13,9 meses sinódicos, a contar do instante $t=0$ registrado na Fig.(9(a)).

\section{Equação para calcular a duração do mês sinódico}

A partir das tabelas de lunações de 1940 a 2020, foram calculados 1001 intervalos de tempo que separam as Luas Novas consecutivas. Cada intervalo de tempo constitui-se em um período sinódico da Lua; a Fig.(7) mostra como esse período se comporta entre 1984 e o final de 2005. Cada mês lunar recebeu um número de ordem (número da lunação), iniciando em 1923. Por exemplo, se consultarmos uma tabela de lunações para 2000, veremos que ela inicia em 6 de janeiro, com a Nova da lunação 953, indo até 25 de dezembro, com a Nova da lunação 965. A Fig.(10) é idêntica à Fig.(7), exceto que, ao invés da data em anos, apresenta o número da lunação.

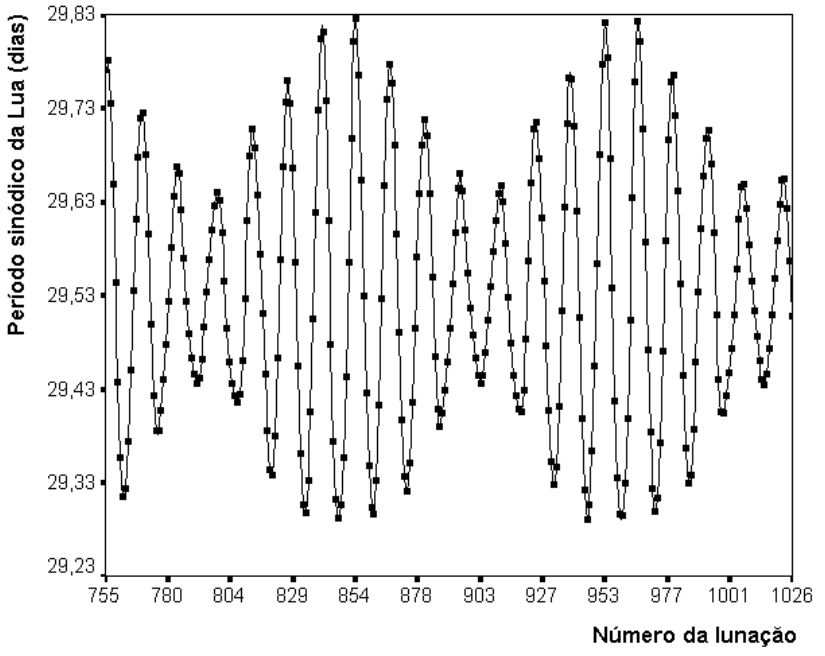

Figura 10. Variação do período sinódico da Lua entre 1984 (lunação 755) e 2006 (lunação 1026).

Com auxílio do pacote estatístico SPSS - Versão 10.0, foi implementada uma regressão não-linear com o objetivo de se encontrar uma equação que permitisse estimar o período sinódico da Lua em função do número $\mathrm{N}$ da lunação. A equação obtida é a seguinte:

$T_{\text {sinódico }}=0,0866 \cos \left[\frac{2 \pi}{12,3689}(N-953)+0,298\right]+$

$$
0,1817 \cos \left[\frac{2 \pi}{13,9444}(N-953)+0,602\right]+29,5306
$$

Nessa equação todos os números expressos com frações decimais são parâmetros obtidos no procedimento de regressão não-linear. A unidade de medida do período sinódico é dia; portanto, as amplitudes das duas funções periódicas e do termo independente da equação são, respectivamente, 0,0866 dia, 0,1817 dia e 29,5306 dias. O erro de cada parâmetro (desvio padrão de cada parâmetro) afeta apenas o último algarismo significativo do respectivo parâmetro. O erro padrão da estimativa (desvio padrão da estimativa) do período é 0,013 dia (19 minutos); os valores extremos do erro da estimativa $^{11}$ nos 1001 períodos sinódicos utilizados no presente estudo (períodos sinódicos entre 1940 e 2020) são -0,031 dia (-45 minutos) e +0,032 dia (+46 minutos).

A Fig.(11) mostra em linha contínua o período sinódico da Lua estimado pela Eq.(1); os pontos são os valores do período sinódico obtido das tabelas de lunações entre 1984 (lunação 755) e o 2006 (lunação 1026). Observa-se que a curva não passa exatamente pelos pontos que representam o período sinódico pois, como referido no parágrafo anterior, a estimativa do período possui erro.

\footnotetext{
${ }^{11} \mathrm{O}$ erro da estimativa ou resíduo é a diferença entre o valor do período predito pela equação de regressão e o valor obtido das tabelas de lunações.
} 


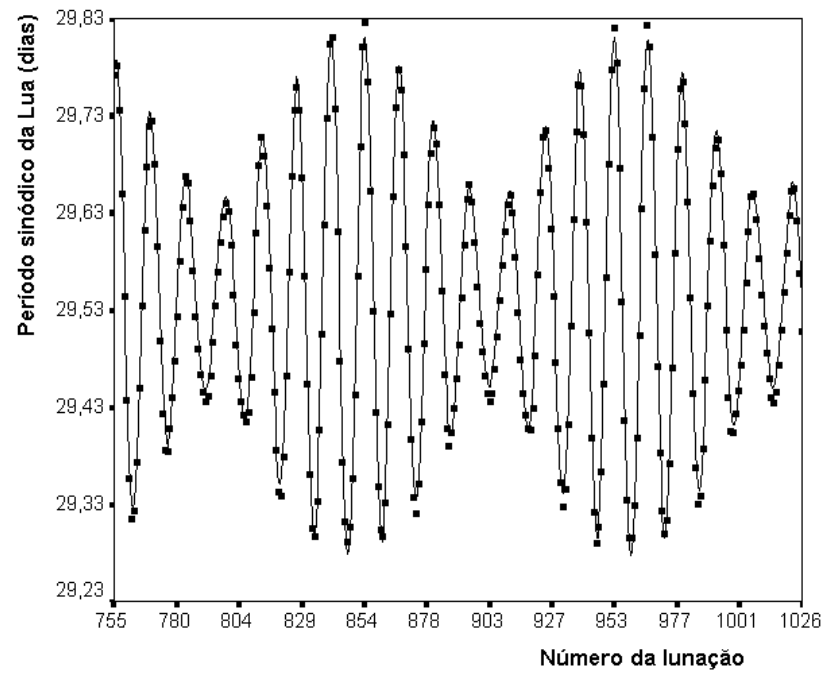

Figura 11. Período sinódico da Lua estimado pela Eq.(1) e obtido das tabelas de lunações entre 1984 (lunação 755) e 2006 (lunação 1026).

O termo independente da Eq.(1) (29,5306 dias) constitui-se no período sinódico médio da Lua. O período da primeira função periódica na Eq.(1) é
12,3689 lunações; como uma lunação dura em média 29,5306 dias, pode-se facilmente calculá-lo em dias: $12,3689 \times 29,5306=365,261$ dias. Esta é a duração do ano e, portanto, a primeira função periódica está relacionada com a perturbação denominada equação anual (vide seção 4). O período da segunda função periódica é 13,9444 lunações ou 1,12738 ano; esse período, conforme o último parágrafo da seção anterior, está relacionado com a perturbação denominada rotação da linha de apside.

\section{Equações para prever as quatro fases principais da Lua}

Novamente, com auxílio do pacote estatístico SPSS Versão 10.0, foi implementada a regressão não-linear com o objetivo de se encontrar equações que permitissem estimar os momentos em que ocorrem cada uma das quatro fases principais da Lua desde 1940 até 2020 inclusive (um total de 4008 fases principais). As equações obtidas são as seguintes ${ }^{12}$ :

$$
\begin{gathered}
t_{\text {Nova }}=0,1723 \cos \left[\frac{2 \pi}{12,3689}(N-953)-1,526\right]+ \\
0,4067 \cos \left[\frac{2 \pi}{13,94436}(N-953)+5,089\right]+29,5305888(N-953)+5,4716, \\
t_{\text {Quarto Crescente }}=0,1723 \cos \left[\frac{2 \pi}{12,3689}(N-953)-1,401\right]+ \\
0,6280 \cos \left[\frac{2 \pi}{13,94436}(N-953)+0,488\right]+29,5305888(N-953)+12,8571, \\
0,4067 \cos \left[\frac{2 \pi}{13,94436}(N-953)+2,172\right]+29,5305888(N-953)+20,2366, \\
t_{\text {Quarto Minguante }}=0,1723 \cos \left[\frac{2 \pi}{12,3689}(N-953)-1,143\right]+ \\
0,6280 \cos \left[\frac{2 \pi}{13,94436}(N-953)+3,856\right]+29,5305888(N-953)+27,6163 .
\end{gathered}
$$

Definindo-se o número $N$ da lunação, as Eqs. (2), (3), (4) e (5), permitem que se calcule um intervalo de tempo em dias que deverá ser adicionado à zero hora (horário normal de Brasília) do dia 01/01/2000 $\left(N_{0}=\right.$ 953). O resultado dessa adição estima o momento da fase principal na lunação $N$. Por exemplo, se especificarmos $N=980$, na Eq.(4) encontraremos $t_{\text {Cheia }}=$ 817,6524 dias. Adicionando esse intervalo de tempo à zero hora de 01/01/2000 encontra-se 15 horas e $39 \mathrm{~min}$ (horário normal de Brasília) do dia 28/03/2002. Se consultarmos a tabela de lunações do Observatório Nacional para 2002, veremos que a Cheia da lunação 980 ocorre em 28/03/2002, às 15 horas e 25 min (portanto, nesse caso estamos estimando o momento da Cheia com erro de apenas $14 \mathrm{~min}$ ).

\footnotetext{
${ }^{12}$ Os números expressos com frações decimais nas Eqs. (2) a (5) são os parâmetros obtidos através do procedimento de regressão não-linear.
} 
O erro de cada parâmetro nas Eqs. (2) a (5) (desvio padrão de cada parâmetro) afeta apenas o último algarismo significativo do respectivo parâmetro. O erro padrão da estimativa (desvio padrão da estimativa) do intervalo de tempo para cada fase principal é 0,014 dia (20 minutos); os valores extremos do erro da estimativa nos 4008 intervalos de tempo para as fases principais utilizados neste estudo são -0,037 dia (-53 minutos) e $+0,035$ dia (+50 minutos). A Fig.(12) é um histograma do erro da estimativa (ou resíduo) com uma curva normal ou gaussiana ajustada; pode-se observar que a distribuição do erro é aproximadamente gaussiana. Uma análise do erro da estimativa ao longo do tempo (1940 a 2020) mostrou não ser possível rejeitar a hipótese de homocedatiscidade; ou seja, o erro da estimativa é homogêneo no tempo.

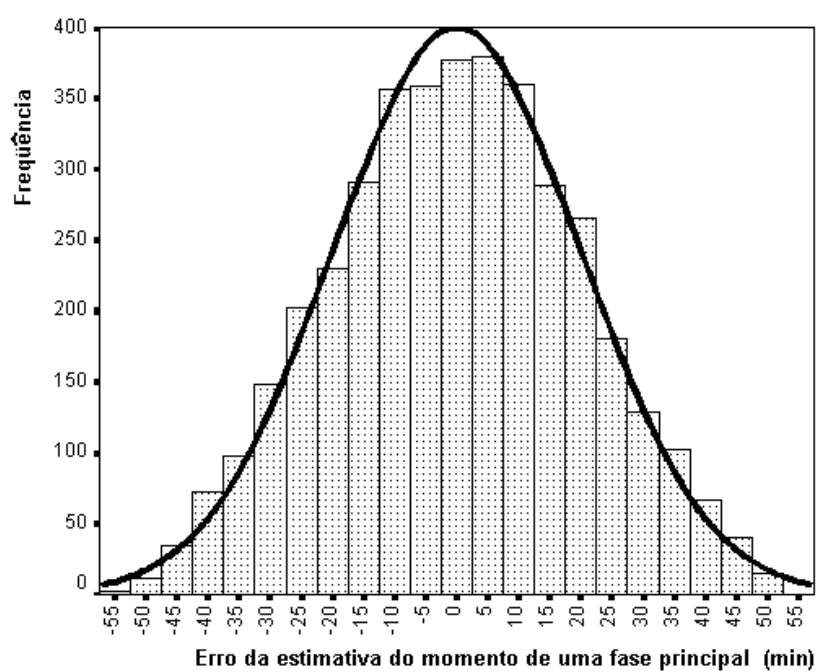

Figura 12. Histograma do erro da estimativa do momento da fase principal.

Utilizando-se as Eqs. (2) a (5) para determinar apenas o dia em que ocorre cada uma das 4008 fases principais deste estudo (isto é, não sendo relevantes os horários dos eventos), verificou-se um acerto de 3953 fases $(98,63 \%)$; em 24 fases $(0,60 \%)$ o erro foi de +1 dia e em 31 fases $(0,77 \%)$ o erro foi de -1 dia.

Da mesma forma como na Eq.(1), as perturbações denominadas equação anual e rotação da linha de apside são reconhecidas nas Eqs. (2) a (5). Adicionalmente, percebe-se que as amplitudes do segundo termo periódico para as Eqs. (2) e (4) são menores do que as das Eqs.(3) e (5); isto é interpretado como decorrente da perturbação denominada variação.

\section{Conclusão}

Neste trabalho aprimoramos a explicação para a ocorrência das fases da Lua em relação à usualmente encontrada em textos introdutórios de Astronomia. Apresentamos qualitativamente as razões mais importantes para a variação no intervalo de tempo que separa duas fases principais consecutivas ${ }^{13}$.

O cálculo do momento de uma fase principal da Lua, com a precisão encontrada nas tabelas de lunações do Observatório Nacional, necessita mais de uma centena termos. Utilizando procedimentos de regressão nãolinear, encontramos equações que permitem estimar o momento de cada uma das quatro fases principais da Lua, no período de 1940 a 2020, com erro padrão de 20 minutos.

\section{Agradecimentos}

Aos professores Rolando Axt, Maria Cristina Varriale, João Batista Harres, Kepler Oliveira Filho e Maria de Fátima Saraiva agradeço a leitura crítica deste trabalho e as sugestões valiosas para o seu aprimoramento.

\section{Referências}

EXPLANATORY Supplement to the Astronomical Ephemeris and the American Ephemeris and Nautical Almanac. Londres: Her Majesty's Stationery Offica, 1977.

LOSEE, J. A historical introduction to the Phylosophy of Science. Oxford: Oxford University Press, 1993.

MARION, J. B. e THORNTON, S. T. Classical dynamics of particles and systems. Fort Worth: Saunders College Publishing, 1995.

MOURÃO, R. R. F. Os eclipses, da superstição à previsão matemática. São Leopoldo: Ed. Unisinos, 1993. OLIVEIRA F⿳, K. S. e SARAIVA, M. F. O. Fundamentos de Astronomia e Astrofísica. Porto Alegre: Instituto de Física da UFRGS, 1997.

PAYNE-GAPOSCHKIN, C. e HARAMUNDANIS, K. Introduction to Astronomy. New Jersey; Prentice-Hall, 1970.

SILVEIRA, F. L. e AXT, R. Questão discursiva número 4 do Provão-2000 do MEC para a Licenciatura em Física. Caderno Catarinense de Ensino de Física, Florianópolis, 17(2): p. 229-234, ago. 2000.

SMART. W. M. Textbook on spherical astronomy. Cambridge: Cambridge University Press, 1979

\footnotetext{
${ }^{13}$ Para maiores detalhes sobre os conceitos astronômicos relevantes ao problema da determinação das fases da Lua pode-se consultar Explanatory Supplement to the Astronomical Ephemeris and the American Ephemeris and Nautical Almanac (1977) ou Smart (1979).
} 